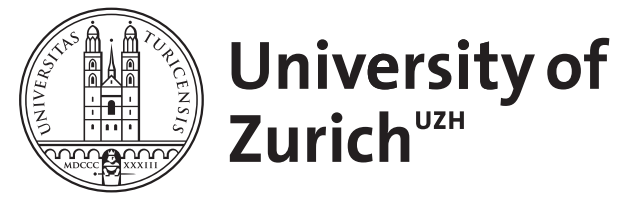

Zurich Open Repository and Archive

University of Zurich

University Library

Strickhofstrasse 39

CH-8057 Zurich

www.zora.uzh.ch

Year: 2016

\title{
Das Kapital nach Marx: Piketty blickt ins 21. Jahrhundert
}

Tanner, Jakob

DOI: https://doi.org/10.7788/ha-2016-0207

Posted at the Zurich Open Repository and Archive, University of Zurich

ZORA URL: https://doi.org/10.5167/uzh-135637

Journal Article

Originally published at:

Tanner, Jakob (2016). Das Kapital nach Marx: Piketty blickt ins 21. Jahrhundert. Historische Anthropologie, 24(2):253-264.

DOI: https://doi.org/10.7788/ha-2016-0207 


\title{
Das Kapital nach Marx
}

\author{
Piketty blickt ins 21. Jahrhundert
}

von Jakob Tanner

Ein Buch als Medienereignis

Thomas Pikettys „Das Kapital im 21. Jahrhundert"1 ist ein Medienereignis. Die Rezeption des Buches wurde durch jene Synergien zwischen Wissenschaft, Politik und persönlicher Prominenz verstärkt, welche die mediale Aufmerksamkeitsökonomie der Gegenwart insgesamt prägen. Pikettys Durchbruch kam 2014 mit der Übersetzung seines voluminösen Werks ins Englische: Während seine seit 15 Jahren vorliegenden, meist zusammen mit andern Autoren verfassten Veröffentlichungen zur Geschichte der Ungleichheit kaum Aufsehen erregten, stieß „Das Kapital im 21. Jahrhundert" in der Anglosphere umgehend auf eminentes Interesse und rief ebenso spontane Zustimmung wie heftige Ablehnung hervor - mit weitreichenden Ausläufern ins Privatleben des Autors. Binnen Kurzem stieg dieser zum „Rock Star“ auf, seine inhaltlich anspruchsvolle Studie erhielt Kultstatus und erstürmte in den Amazon-Charts den Spitzenplatz. ${ }^{2}$ Im Prisma des medialen Rampenlichts schien in Pikettys Persönlichkeit ein breites Spektrum von Wertungen auf: vom genialen „,neuen Marx“ und unprätentiösen Hochleistungsforscher, über den telegenen Meisterdenker und populären Thesenlieferanten bis hin zum maliziösen „Neidforscher“ und ,woman beater". 3

Offensichtlich hatte das Buch einen Nerv der Zeit getroffen. Es löste zudem massive Erschütterungen in der intellektuellen Tektonik der Wirtschaftswissenschaften aus. Während US-amerikanische Nobelpreisträger wie Paul Krugman, Joseph Stiglitz und Robert Solow einen epochalen Durchbruch für die Wirtschaftswissenschaften feier-

1 Thomas Piketty, Das Kapital im 21. Jahrhundert. Übersetzt aus dem Französischen von Ilse Utz und Stefan Lorenzer, München 2014. (Originalausgabe: Le capital au XXIe siècle, Paris 2013). Die Seitenangaben beziehen sich auf die deutsche Ausgabe.

$2 \mathrm{Vgl}$. http://nymag.com/daily/intelligencer/2014/04/on-tour-with-rock-star-economist-thomaspiketty.html\# (01.05.2016).

3 „Daily Mail“, „National Post“ und weitere rechtsstehende Boulevardblätter berichteten ab Frühjahr 2014 unter diesem Stichwort von einem später eingestellten Verfahren, das Aurélie Filippetti, zwischen Mai 2012 und August 2014 französische Kulturministerin, im Jahre 2009 als Pikettys Ex-Partnerin angestrengt hatte: http://www.dailymail.co.uk/news/article-2 619675/The-Lefts-rockstar-economist-Thomas-Piketty-woman-beater-says-former-lover.html\#ixzz31bWZsaxV. Zu den Reaktionen in der französischen Presse vgl. den Bericht „Piketty, entre l'ombre et la lumière“ in der kanadischen Tageszeitung „La Presse“ vom 9. Mai 2014 (http://www.lapresse.ca/international/ europe/201 405/09/01-4 765 120-piketty-entre-lombre-et-la-lumiere.php). 
ten, setzten andere Fachvertreter auf Demontage. In den Feuilletons großer deutscher Tageszeitungen hagelte es Verrisse und auch aus andern Weltgegenden kam Kritik. ${ }^{4}$ Eine ganze Reihe von Rezensenten stellte Pikettys Datenbasis und sein Erklärungsmodell in Frage, andere - wie etwa Hernando de Soto - rügten die einseitige Ausrichtung der Studie auf die reichen Industrieländer und äusserten den Vorwurf eines ,armseligen Eurozentrismus“. 5

Tatsächlich bleiben trotz verstreuter Ausführungen zur „weltweiten Verteilung der Produktion“ (88-93) und zur „weltweiten Ungleichheit“ (94-101) viele Regionen des Globus in Pikettys Studie ausgespart oder unterbelichtet. Und auch bewundernde Kommentare bemängeln, der Autor stelle die Wertsteigerungsdynamik des Kapitals zu einfach dar; vor allem habe er die spezifische Rolle des Immobilienvermögens und der stetig steigenden Bodenpreise übersehen. Doch der Hauptaussage der Studie, dass die Einkommens- und Vermögensunterschiede in den reichen Industriestaaten seit den 1970er Jahren in signifikantem Ausmaß zugenommen haben, vermochte diese Kritik wenig anzuhaben.

Piketty mobilisiert ein eindrückliches Statistikarsenal für seine Beweisführung. Aus der internationalen Zusammenarbeit mit 30 Forschern ging die World Top Incomes Database hervor, die für fast zwei Dutzend wirtschaftlich entwickelter Länder Aufschluss gibt über die Reichtumskonzentration im obersten Prozent und - noch ausgeprägter - im obersten Promille der gesellschaftlichen Sozialpyramide. ${ }^{6}$ Das hervorstechende Phänomen ist der Aufstieg der Supermanager und der Höhenflug ihrer Gehälter. Das Argument, dies sei einer ebenso rasch wachsenden Grenzproduktivität in diesem Segment geschuldet, hält Piketty bestenfalls für naiv. Viel plausibler erklärt er die Lohnexplosion mit dem „Handgriff in die Kasse“ und mit der fiskalischen Privilegierung von Spitzenverdienern. Die sich verhärtenden Strukturen der Kapitalmacht lassen sich allerdings auch in anderen Bereichen beobachten. Sie lassen sich nicht auf psychologische Motive wie etwa eine „Profitgier“ zurückführen, sondern resultieren aus einer kapitalistischen Akkumulationslogik, welche eine moderne, aufgeklärte, egalitären Normen verpflichtete Gesellschaft systematisch unterminiert - es sei denn, die Demokratie verteidige ihre Grundlagen mit wirksamen politischen Maßnahmen.

\section{Die Rückkehr der Verteilungsfrage}

Thomas Pikettys Messlatte hängt hoch. Schon mit dem Titel positioniert sich das 800-seitige Buch als Opus magnum, sind doch die Assoziationen zu Karl Marx' Hauptwerk „Das Kapital“ von 1867 unverkennbar. Piketty schildert, was mit dem Kapital passiert ist, seit Marx vor fast 150 Jahren die „Dynamik eines sich voll ent-

4 Einen Überblick auf die Debatte von 2014 gibt Julian Bank, Schwache Argumente? Einblicke in den Abgrund der deutschen Piketty-Debatte, veröffentlicht am 30. Juni 2014 auf der InternetPlattform ,,verteilungsfrage.org - Zur Politik und Ökonomie der Ungleichheit“: http://verteilungsfrage.org/2014/06/schwache-argumente-einblicke-in-den-abgrund-der-deutschen-piketty-debatte/ (25.05.2016).

5 Hernando de Soto, Pikettys armseliger Eurozentrismus, in: Neue Zürcher Zeitung, 05. 02. 2015.

6 http://www.wid.world/ (24.05.2016). 
wickelnden Industriekapitalismus" (20) analysiert hat. Er konstatiert eine frappante Parallele: „In gewisser Weise sind wir zu Beginn des 21. Jahrhunderts in der gleichen Situation wie die Beobachter des 19. Jahrhunderts“ (32). Das Kapital hat seine Bedeutung alles andere als eingebüsst, ,nur seine Form hat sich gewandelt: was damals [im 18. und noch weit ins 19. Jahrhundert hinein, JT] der Landbesitz war, ist heute das Immobilien-, Industrie- und Finanzkapital“ (501). Ebenso wie die Jahrzehnte zwischen 1870 und 1914 (d.h. die Phase der ,,ersten Globalisierung der Finanzund Wirtschaftsströme“) ist auch die „Zweite Globalisierung“ seit den 1970er Jahren durch eine ,immense Ungleichheit“ (49) charakterisiert.

Das Verbindende zwischen Piketty und Marx ist der Problemzugang: ,Wir werden [...] sehen, dass die Marx'sche Analyse trotz ihrer Grenzen in mehreren Punkten noch immer relevant ist.“ Denn Marx gehe ,,von einer echten Frage aus (einer unglaublichen Konzentration von Vermögen während der Industriellen Revolution)“ (25). Darauf richtet auch Piketty sein Augenmerk und unterstützt markant jene Bestrebungen, die nach einer langen Phase einer ausgeprägten Geschichtsvergessenheit und Machtblindheit der Wirtschaftswissenschaften auf die eminent politische Verteilungsfrage zurückkommen wollen. Im beginnenden 21. Jahrhundert lässt sich diese Frage allerdings nicht mehr auf einzelne Wissenschaftsdisziplinen herunterbrechen. Sie sei, so Piketty, ,zu wichtig, um sie allein den Ökonomen, Soziologen, Historikern und Philosophen zu überlassen“ (14). Deshalb müssten Film, Literatur und Kunst in die Analyse einbezogen werden.

Insbesondere der Roman des 19. Jahrhunderts liefert aus Pikettys Sicht ein präzises Bild der Machtbeziehungen, Karrieremuster und Vermögensverhältnisse in der sich formierenden bürgerlich-kapitalistischen Gesellschaft und vermittelt einen unbestechlichen Einblick in die „Tiefenstrukturen des Kapitals“ (13). Für die vier Jahrzehnte zwischen 1790 und 1830 schildern Jane Austen (für England) und Honoré de Balzac (für Frankreich) die ,ihre Gesellschaften prägenden Vermögenshierarchien“: „Sie spüren ihren verborgenen Grenzen nach und wissen um die unerbittlichen Folgen für das Leben dieser Männer und Frauen, um ihre Bündnisstrategien, ihre Hoffnungen und ihr Unglück“ und liefern so wahrhaftig-eindringliche Beschreibungen, ,die keine Statistik und keine wissenschaftliche Analyse zu bieten vermag“ (14). In Balzacs Roman Père Goriot aus dem Jahre 1835 wird die Geschichte einer ,vom Geld durch und durch korrumpierten Gesellschaft" erzählt; die Interaktionen der Hauptprotagonisten bewegen sich durchwegs im Gravitationsfeld eines Reichtums, der auf die Arbeit pfeift. In spannungsreichen Szenen werden alle schönen Illusionen, man könne es in einem solchen System ,durch Studium, Verdient und Arbeit“"(316) zu etwas bringen, schonungslos zum Platzen gebracht. Angesichts der Verschmelzung einer Managerund einer neuen Rentnerklasse im globalen Patrimonialkapitalismus des 21. Jahrhunderts sind diese Schilderungen von beklemmender Aktualität.

Pikettys Darstellung bricht aus einer sterilen Gegenüberstellung von quantitativen und qualitativen Ansätzen, von dünner Analyse und dichter Beschreibung, von Zählen und Erzählen aus. Der Autor verbindet die Wertschätzung von literarischen und visuellen Quellen mit einem Hang zur empirischen Kärrnerarbeit und einem innovativen Umgang mit formalen Erklärungsmodellen. Die Referenz, die er Marx erweist, provoziert zugleich eine dreifache Kritik. Erstens habe Marx ,trotz all seiner wich- 
tigen intuitiven Annahmen die verfügbaren Statistiken nicht systematisch ausgewertet“, er nehme nirgends Bezug ,auf die Versuche [...], den nationalen Kapitalstock zu schätzen“, und scheine „,die entstehende volkswirtschaftliche Gesamtrechnung völlig außer Acht gelassen zu haben“ (304). Da erscheint ein Marx, der mehr theoretischer Träumer als empirischer Schwerarbeiter war und der in einer ,politisch aufgeheizten Atmosphäre" (24) sein statistisches Banausentum mit jenen apokalyptischen Prognosen zu kombinieren verstand, die fortan ebenso die Prognose eines Untergangs des Kapitalismus wie den unentwegten Kampf für den Sozialismus plausibilisierten. Pikettys zweiter und dritter Punkt, die sich auf theoretisch-konzeptionelle und letztlich politische Fragen beziehen, werden in den folgenden zwei Abschnitten ausgeführt. Es geht dabei (zweitens) um das Verhältnis von Klassen- und Demokratietheorie und (drittens) um den epistemologischen Anspruch sowie die Erwartungshaltung, die sich mit der Kapitalanalyse verbinden.

Demokratie und Arbeit

Als Theoretiker der kapitalistischen Produktionsweise und der bürgerlichen Klassengesellschaft interessierte sich Marx für den Antagonismus und die Widersprüche in den Beziehungen zwischen Kapital und Arbeit. Durch alle ihre facettenreichen Epiphänomene hindurch lässt sich die Geschichte aus der Dynamik der Klassenkämpfe heraus entziffern. Demokratie hat in einem solchen Ansatz ebenso wenig einen Eigenwert wie bürgerliche Freiheiten, ein parlamentarisches System oder ein Rechtsund Verfassungsstaat. Marx entwirft eine Arbeitswert- und Mehrwerttheorie, welche die Arbeit als exklusiv wertschöpfendes Prinzip begreift und damit gesellschaftspolitisch nobilitiert. Die ganze menschliche Geschichte erscheint als Selbsthervorbringung des Menschen durch seine Arbeit und mit dem Kapitalismus hat diese einen historischen Entwicklungsstand erreicht, der nun den Übergang in eine herrschaftsfreie Vergesellschaftung ermöglicht. Mit dieser Metaphysik der Arbeit korrespondiert die Kritik am Ausbeutungsmodus von Klassengesellschaften. Mit der kapitalistischen Fabrikindustrialisierung wird die Entäusserung in der Arbeit und - als deren Kehrseite - die Aneignung von Wert durch das Kapital auf eine neue Stufe gehievt. Die mechanisierungsgetriebene Produktionssteigerung verdrängt die absolute durch die relative Mehrwertproduktion. Trotz der Verbesserung der materiellen Lebensbedingungen der arbeitenden Bevölkerung nehmen Menge und Macht, zugleich aber auch die Verwertungsschwierigkeiten des Kapitals zu. Der kapitalistische Akkumulationsprozess kann nicht anders als krisenhaft verlaufen; auf lange Sicht wird er durch das Gesetz des tendenziellen Falls der Profitrate unterminiert.

Für Marx löst sich die Demokratiefrage in der Dynamik der kapitalistischen Produktionsweise auf. Die mit dem Fabrikkapitalismus aufkommende „grosse Industrie“ setzt zwingend eine durch Konkurrenz und Kredit vorangetriebene Tendenz zur Zentralisation frei. ${ }^{7}$ Die Logik der Kapitalakkumulation ,erzeugt mit der Notwendigkeit

7 Karl Marx, Das Kapital (1867), Marx-Engels-Werke (MEW) Bd. 23, Berlin 1972, 655. 
eines Naturprozesses ihre eigene Negation“: „Die Zentralisation der Produktionsmittel und die Vergesellschaftung der Arbeit erreichen einen Punkt, wo sie unverträglich werden mit ihrer kapitalistischen Hülle. Sie wird gesprengt. Die Stunde des kapitalistischen Privateigentums schlägt. " 8 Weil die Kapitalisten ihre Herrschaftsposition aber nicht freiwillig aufgeben, erscheint ,die Klassendiktatur des Proletariats als notwendiger Durchgangspunkt zur Abschaffung der Klassenunterschiede überhaupt“. ${ }^{9}$ Politische Gewalt erhält den Charakter einer „ökonomischen Potenz“. Schlüssig kritisiert Marx die „Freiheitsillusion“ der bürgerlichen Gesellschaft und lässt keinen Zweifel an der schließlichen „Eroberung der politischen Gewalt durch die Arbeiterklasse“. ${ }^{10}$ Die proletarischen Schlachtrufe „Expropriiert die Expropriateure“, „Arbeiter aller Länder vereinigt euch“ und „Die Befreiung der Arbeiterklasse kann nur das Werk der Arbeiter selber sein" leiten sich aus einer solchen Konzeption ab. Sie liess sich aber, insbesondere mit dem Postulat einer „Klassendiktatur“, auch in eine Legitimationsideologie für Staatsbürokratien umschreiben, die ihre Gewaltherrschaft im Namen eines „Proletariats“ oder der ,arbeitenden Massen“ ausübten.

Für Marx waren solche Perversionen aufgrund seiner Geschichtsteleologie schlicht nicht denk- oder vorstellbar. ${ }^{11}$ Demokratietheoretisch verblieb er im Horizont seiner Frühschriften, in denen er geschrieben hatte, die Demokratie sei „das aufgelöste Rätsel aller Verfassungen“, weil hier die Entfremdung von Mensch und Staat aufgehoben sein würde. Dieselbe Formulierung verwendete er austauschbar für den Kommunismus als „das aufgelöste Rätsel der Geschichte“, das sich als dessen Lösung weiss. ${ }^{12}$

Sowenig sich in einem solchen Denkansatz eine Grundlagenreflexion über die institutionellen und rechtlichen Bedingungen einer demokratischen Gesellschaft entfalten konnte, so sehr war er geeignet, das Sensorium für den Arbeitsprozess und den „Formwechsel der Arbeit“" zu steigern. ${ }^{13}$ Marx löst die Makrostruktur des Kapital-Arbeit Antagonismus in plastische Mikrogeschichten der kapitalistischen Aneignung der Arbeit auf. Er schildert minutiös den Aufstieg der ,abstrakten menschlichen Arbeit“" und das „produktive Tun des menschlichen Körpers“. Die „,moderne Industrie [...] revolutioniert [...] beständig die Teilung der Arbeit im Innern der Gesellschaft und schleudert unaufhörlich Kapitalmassen und Arbeitermassen aus einem Produktionszweig in den andern. Die Natur der grossen Industrie bedingt daher Wechsel der Arbeit, Fluss der Funktion, allseitige Beweglichkeit des Arbeiters." 14

Schon in vielen Vorarbeiten zum „Kapital“ zeigt Marx, wie sich die Ausbeutung in den menschlichen Körper einschreibt. Die reale Subsumption der Arbeit unter das Kapital erweist sich als ,ununterbrochenes Opferfest der Arbeiterklasse“, in dem die „Ungeheuerlichkeit einer elenden, für das wechselnde Exploitationsbedürfnis des Ka-

8 Ebd., 791.

9 Karl Marx, Die Klassenkämpfe in Frankreich (1850), in: MEW Bd. 7, Berlin 1973, 89.

10 Marx, Das Kapital, 779, 562, 512.

11 Vgl. dazu auch die aufschlussreiche Präzisierung von Karl Marx an den Redaktor der „Neue Deutsche Zeitung“ Nr. 158 vom 4. Juli 1850. in: MEW Bd. 7, Berlin 1973, 323-324.

12 Karl Marx, Zur Kritik der Hegelschen Rechtsphilosophie 18343/44, in: MEW Bd. 1, Berlin 1976, 231; Karl Marx, Ökonomisch-philosophische Manuskripte (1844), in: MEW, Ergänzungsband, 1. Berlin 1968, 536.

13 Marx, Das Kapital (1867), MEW Bd. 23, 58.

14 Ebd., 61, $510 \mathrm{f}$. 
pitals in Reserve gehaltenen, disponiblen Arbeiterbevölkerung“ ersetzt wird „durch die absolute Disponibilität des Menschen für die wechselnden Arbeitserfordernisse“ ${ }^{15}$ Im Wissen und der Wissenschaft sieht Marx ein entscheidendes Drehmoment dieser kapitalistischen Umwandlung des Produktionsprozesses. Mit fortschreitender Mechanisierung treten den Arbeitern ihre eigenen Produkte als Kapital gegenüber: „In der Maschinerie tritt die vergegenständlichte Arbeit der lebendigen Arbeit [...] als sie beherrschende Macht gegenüber"; die kapitalistischen Produktivkräfte sind „,vergegenständlichte Wissenschaft“ und ,die Entwicklung des capital fixe zeigt an, bis zu welchem Grade das allgemeine gesellschaftliche Wissen, knowledge, zur unmittelbaren Produktivkraft geworden ist“.${ }^{16}$ Und die ,ganz moderne Wissenschaft und Technologie“ transformiert die „,buntscheckigen, scheinbar zusammenhangslosen und verknöcherten Gestalten des gesellschaftlichen Produktionsprozesses [...] in bewusst planmässige [...] Anwendungen der Naturwissenschaft".${ }^{17}$

Knapp 150 Jahre nach dem Erscheinen von „Das Kapital“ finden sich in Pikettys Analyse gegenläufige Sensibilitäten. „Das Kapital im 21. Jahrhundert“ liest sich weitgehend als Gegenstück zur Marx'schen Darstellung. Weil Piketty die Arbeitswerttheorie ad acta gelegt hat und den kapitalistischen Akkumulationsmechanismus nicht als Wertproduktion durch Arbeit entziffert, kann er die Begriffe „Vermögen“, „Reichtum“ und „Kapital“ synonym verwenden. Ebenso amorph bleibt der Begriff der Arbeit. Arbeiterinnen und Arbeiter geraten primär als Staatsbürger und - unter dem Aspekt der Einkommen - als Konsumenten ins Blickfeld (wobei die Geschlechterdimension weitgehend ausgeklammert wird). Arbeitsprozesse bleiben unsichtbar, weil sich die Aufmerksamkeit von der Herrschaft des Kapitals über die Arbeit auf das Spannungsfeld zwischen Demokratie und Ungleichheit verlagert. Für Piketty steht die Frage im Zentrum, ob und wie ,die Demokratie eines Tages die Kontrolle über den Kapitalismus zurückgewinnen“ kann (784).

Die Macht des Kapitals analysiert er mittels zweier „fundamentaler Gesetze“. Erstens kann die Kapitalrendite (r) langfristig über der Wachstumsrate von Produktion und Einkommen (g) liegen - und tut dies auch, obwohl es Gegenkräfte gibt, die vor allem im allgemeinen Zugang zu Wissen und Bildung angelegt sind. Dieses ökonomische Gesetz wirkt nicht fatalistisch - Piketty lehnt einen ökonomischen Determinismus explizit ab und fasst den Möglichkeitsraum der Politik ins Auge. Hier gibt es allerdings keine Allianzen für Gegenmaßnahmen, so dass die Ungleichung $r>g$ ungebremst wirkt und „,massive Divergenzkräfte“ generiert, ,die unsere demokratischen Gesellschaften und jene soziale Gerechtigkeit bedrohen, die zu ihren Legitimationsgrundlagen zählen“ (785). Das zweite Gesetz bezieht sich auf das „Kapital-Einkommens-Verhältnis“" (ß) einer Volkswirtschaft. Diese Kennziffer $ß$ gibt Auskunft darüber, wie hoch über längere Zeiträume hinweg der Gesamtwert aller Vermögen im Verhältnis zum Nationaleinkommen ist. Lag dieser Wert für reiche Länder und bezogen auf die (vorherrschenden) Privateinkommen Anfang der 1970er Jahre beim Faktor 2 bis

15 Ebd., $511 \mathrm{f}$.

16 Karl Marx, Grundrisse der Kritik der politischen Ökonomie (1857/58), Frankfurt a. M. - Wien (o. J.), 585, 594.

17 Marx, Das Kapital (1867), 510. 
3,5 , so hat er sich vierzig Jahre später verdoppelt und erreicht wiederum Werte wie in den Jahren um 1900.

Diese „Rückkehr des Kapitals in den reichen Ländern seit den 1970er Jahren“ (226) lässt sich erklären, wenn B als Quotient von Sparquote (s) und der Wachstumsrate (g) dargestellt wird $(B=\mathrm{s} / \mathrm{g})$. Wenn viel gespart wird und das Wirtschaftswachstum verhalten ausfällt, so wächst der Kapitalstock gewaltig an. Die Tendenz wird verstärkt, weil bei zunehmender Ungleichheit mehr Reiche mehr sparen. Anders ausgedrückt: „In einer nahezu stagnierenden Gesellschaft gewinnen die in der Vergangenheit angesammelten Vermögen eine unverhältnismäßig grosse Bedeutung “ (220). In diesem Zusammenhang weist Piketty die Hypothese eines „Aufstiegs des Humankapitals“ (40) zurück; die Subsumtion der durch Qualifikation erworbenen Fähigkeiten unter dem Kapitalbegriff erscheint ihm gerade markttheoretisch überhaupt nicht plausibel. Während Sklaven, die auf freien Märkten gekauft und verkauft werden können, dem Kapitalbestand zugerechnet werden müssen, gilt dies für Lohnarbeit gerade nicht (213ff).

Das Argument bleibt auch hier systematisch und Piketty geht nirgends auf die qualitativen Veränderungen der Arbeit durch Automatisierungsprozesse, durch neue Qualifikationsroutinen sowie Bildungsangebote ein. Soweit die Relation von Kapital und Einkommen anhand von Quellen wie den erwähnten Romanen en détail geschildert wird, geht es um Karrieremuster und Aufstiegsbarrieren - und nicht um Bedingungen der Arbeit und den Alltag arbeitender Menschen. Es dominiert durchwegs eine demokratietheoretische Fragestellung; so wird die Ausbeutungsproblematik weitgehend unter dem Aspekt der Armut (und ihrer Bekämpfung) behandelt.

Entsprechend negativ fällt die Interpretation der Sowjetunion aus. Lenin kommt im ganzen Buch nicht vor; die Russische Revolution ist in ihrem Anspruch fundamental gescheitert. Piketty spricht von ,menschlichen Desastern, die von der zentralen Planwirtschaft heraufbeschworen wurden“ (723). Im Kontrast dazu qualifiziert er konsequenterweise den ,sozialdemokratischen Weg“, den die fortgeschrittenen Länder - im Kontrast zur Sowjetunion - beschritten haben, als einen „Glücksfall“ für ihre Bevölkerungen (24). Denn die Sozialdemokratie hat verstanden, dass sich „,die Funktion des Privateigentums und der Marktwirtschaft nicht darin (erschöpft), die Herrschaft der Kapitalbesitzer über diejenigen zu sichern, die nichts außer ihrer Arbeit haben“. Im Unterschied zur „totalitären Versuchsanordnung“ des „sowjetischen Staates“, dessen Planungsbeauftragte weitgehend im Nebel stocherten (774), hat der Reformsozialismus realisiert, dass kein Weg an marktwirtschaftlichen Koordinationsmechanismen vorbeiführt - und zwar aus demokratietheoretischen Überlegungen. Generell seien ,die parlamentarischen Einrichtungen und der Rechtsstaat [...] nicht einfach die bourgeoisen Institutionen, als die sie von den marxistischen Intellektuellen vor dem Mauerfall beschrieben werden“ (791). Konsequenterweise sieht Piketty sein Buch nicht als Anleitung für eine Kampfstrategie der Arbeiterklasse, sondern als „einen bescheidenen Beitrag zur Bestimmung der sozialen Organisationsformen, Institutionen und Politiken [...], die am besten geeignet sind, eine gut funktionierende gerechte Gesellschaft zu schaffen, und zwar im Rahmen eines Rechtsstaates, dessen Regeln bekannt sind, für alle gelten und demokratisch diskutiert werden können“ (52). 
Illusionen des Kalten Krieges und Niedergangsprognosen

Piketty lehnt die - wie er sie nennt - ,Vorliebe für apokalyptische Prognosen“ (25) bei frühen Ökonomien (wie David Ricardo) und im Marx'schen Theorieentwurf ab. Mit dem Gesetz $r>g$ hebelt er - und das ist der dritte Punkt - das Gesetz des tendenziellen Falls der Profitrate aus. Aus seiner Sicht besteht das Problem nicht darin, dass Kapitalismus dereinst an seinen eigenen Widersprüchen zerbricht, sondern dass er die Demokratie zerstört. Anders als Marx fasst er die kapitalistische Produktionsweise als äußerst anpassungsfähig auf. So war die rasche Expansion des Finanzkapitalismus' seit den 1970er Jahren an der Steigerung der Kapitalrendite beteiligt. Generell ermöglicht es der Trend zur Verbilligung neuer Technologien, die Profitrate nachhaltig und langfristig hoch zu halten.

Piketty weist immer wieder darauf hin, dass die Politik diesen Tendenzen einen Riegel vorschieben kann und dass die historische Entwicklung des Kapitalismus deshalb kontingent ist. Er betont, dass die der Kapital-Arbeit-Antagonismus in der Vergangenheit durch gesellschaftliche Erschütterungen nachhaltig umgeformt wurde. Der Erste Weltkrieg, der sich nahtlos an die Belle Époque anschloss, unterhöhlte die obszöne Macht des Kapitals. In den Jahren 1914 bis 1918 zerstörte die Vernichtungsmaschine eines auf großindustrieller Stufenleiter bis zur wirtschaftlichen Erschöpfung geführten Krieges sehr viel Kapital. In der Weltwirtschaftskrise und im Zweiten Weltkrieg setzte sich dieser Prozess fort. Trotz des sich während der Trente glorieuses (zwischen 1945 und 1975) ausbreitenden Glaubens an eine immerwährende Prosperität stand dieser Zeitraum im Schatten des vorangehenden Katastrophenzeitalters.

Dieser düstere Hintergrund konnte jedoch verdrängt werden, weil das Gleichgewicht des Schreckens- in Ost und West - mit hochfliegenden Hoffnungen koexistierte. In der „freien Welt" beflügelte die Aussicht auf die demokratische Domestizierung des Kapitalismus sozialstaatliche Umverteilungsprojekte und eine antizyklische Konjunkturpolitik (durch eine keynesianische Globalsteuerung der volkswirtschaftlichen Gesamtnachfrage). Die schroffen Klassengegensätze, die sich im 19. Jahrhundert mit der Fabrikindustrialisierung vertieft hatten, wichen einer ausgeglicheneren Einkommensverteilung. Die Lohnschere begann sich, zunächst noch unmerklich, zu schließen. Fatale Wirtschaftsdepressionen milderten sich zu konjunkturellen Rezessionen ab. Das Kapital erschien vor allem in Form der mechanisierten Massenproduktion, welche das Pro-Kopf-Einkommen in bisher unbekanntem Ausmaß steigerte und eine nie dagewesene Kaufkraftsteigerung in der ganzen Breite der Gesellschaft ermöglichte. Der Arbeitsmarkt integrierte zunehmend Frauen, die Arbeitsmigration nahm zu. Bald war von einer Massenkonsum- und Freizeitgesellschaft die Rede, in der einstige Statusgüter, insbesondere das Automobil, an der Basis der Sozialpyramide ankamen. Die politische Demokratie schien gleichsam ihr marktwirtschaftliches Pendent erhalten zu haben. Für den Westen war dies im Systemwettbewerb mit dem Osten eine beruhigende Erfahrung, die nahelegte, in Marx einen veralteten Autor aus dem 19. Jahrhundert zu sehen.

Der amerikanische Wirtschaftshistoriker Simon Kuznets brachte den Rückgang der Ungleichheit Mitte der 1950er Jahre mit seiner umgekehrten U-Kurve visuell einprägsam auf den Punkt. Das moderne Wirtschaftswachstum sei - so seine These - in einer 
ersten Phase aufgrund der zunehmenden sozialen Kluft zwischen den Klassen durch harte politische Konflikte charakterisiert, um dann aber, mit der Steigerung der Produktivität und der Verallgemeinerung des Wohlstandes, in eine egalitäre Ära mit Chancengleichheit und ausgeglichenen materiellen Lebensbedingungen überzugehen. Dieses „Produkt des Kalten Krieges“, das sich seit den 1960er Jahren auch mit dem neu aufkommenden Schlagwort einer für alle zugänglichen „Wissensgesellschaft“ paarte, wird von Piketty hart kritisiert. Zum einen sei „,die märchenhafte Theorie der ,Kuznets-Kurve“ weitgehend aus fragwürdigen Gründen“ und ,auf dürftiger empirischer Basis formuliert“ worden (31). Der Wille zur Verbreitung „guter Nachrichten“ sei hier Vater des Gedankens gewesen. Zum anderen sei die von Kuznets verabsolutierte Tendenz gerade nicht durch eine gewaltfreie demokratische Rationalität in Gang gesetzt worden, sondern ein Resultat von Krieg und Krisen (362) gewesen. Aus der Tatsache, dass gesellschaftliche Katastrophen zwischen 1914 und 1945 die Macht des Kapitals reduziert hätten, lässt sich kein Optimismus schöpfen. Vielmehr regenerieren die Vermögenden ihr Herrschaftspotenzial längerfristig gerade unter demokratischfriedlichen Bedingungen.

Werden aus überzeugten Sozialdemokraten ,widerwillige Radikale“?

Piketty argumentiert sich so in eine paradoxe Situation hinein. Er würdigt die Wachstums- und Konsumleistung des Kapitalismus, stellt aber fest, dass aufgrund des Gesetzes $r>g$ (d.h. die Kapitalrendite ist höher als die wirtschaftliche Wachstumsrate) ,,automatisch inakzeptable und willkürliche Ungleichheiten“ entstehen, ,die das Leistungsprinzip, auf dem unsere demokratischen Gesellschaften basieren, radikal infrage stellen“ (14). Und er hält an der ,unersetzlichen Rolle“ eines „Preissystems“ (und damit eines marktwirtschaftlichen modus operandi) zur „Koordinierung der Handlungen von Millionen - ja Milliarden von Menschen im Rahmen der neuen Weltwirtschaft“ (19) fest, geht aber gleichzeitig davon aus, dass eine gut funktionierende Marktwirtschaft keine Konvergenzkräfte freisetzt, sondern dass es gerade „,perfekte“ Kapitalmärkte sind, welche die Macht des Kapitals befestigen (47, 500). Die „Rückkehr des Kapitals“ ist im 21. Jahrhundert deswegen so massiv, weil der Kapitalismus keineswegs an einer Profitklemme leidet, sondern sich zugunsten des Kapitals auf globaler Stufenleiter zu reorganisieren vermochte.

Welche politischen Konzepte und Strategien können unter diesen Bedingungen weiterhelfen? Die Verklärung Pikettys zu einem ,neuen Marx“ und die teilweise wüste Kritik an seinem linken Weltbild dürfen nicht über den theoretisch-politischen Konformismus hinwegtäuschen, der seiner Analyse zugrunde liegt. Die Manchester Soziologin Miriam Ronzoni hat vor kurzem unter dem Titel „How social democrats may become reluctant radicals" auf Parallelen zwischen Thomas Piketty und Wolfgang Streeck hingewiesen. ${ }^{18}$ Von sozialdemokratischen Überzeugungen ausgehend, stellen

18 Miriam Ronzoni, How social democrats may become reluctant radicals: Thomas Piketty's Capital and Wolfgang Streeck's Buying Time, Review-Article in: European Journal of Political Theory (2015) 1, 1-10. 
beide Autoren fest, dass sich Kapitalismus und Demokratie nur unter spezifischen historischen Bedingungen vereinbaren lassen. Heute sind diese nicht mehr gegeben. Was also tun? Piketty insistiert darauf, er sei aufgrund seiner Lebenserfahrung „,immun gegen die herkömmlichen und wohlfeilen antikapitalistischen Diskurse" und angesichts des ,gewaltigen historischen Scheiterns“ des Ostblocks liege ihm nichts daran, „den Kapitalismus als solchen zu kritisieren“ (52). Sein wissenschaftlicher Wahrheitsanspruch ist agnostisch unterfüttert: „Marx wollte absolute Wahrheit - ich nicht“, erklärte er in einem Interview. ${ }^{19}$

Die wichtigste Gegenkraft gegen die Aushöhlung der Demokratie durch Marktimperative und Kapitalmacht sieht Piketty nicht (wie Streeck) im Nationalstaat, sondern in transnationalen und globalen Lösungsansätzen. So plädiert er für eine institutionelle Vertiefung des europäischen Integrationsprozesses und für „eine Demokratie im europäischen Maßstab“ (788). ${ }^{20}$ Daraus ergibt sich durchaus logisch, dass Piketty - auch hier sozialdemokratisch grundiert - für ein meritorisches Weltbild eintritt. Als Eingangsmotto zu seiner Studie zitiert er Artikel 1 der Erklärung der Menschenund Bürgerrechte von 1789: „Gesellschaftliche Unterschiede dürfen nur im allgemeinen Nutzen begründet sein." Soziale Ungleichheit stelle „an sich kein Problem dar [...], wenn sie auch nur im Geringsten gerechtfertigt ist" (52). In jeder Demokratie stehe ,die proklamierte Gleichheit der Bürgerrechte in eklatantem Kontrast zur faktischen Ungleichheit der Lebensbedingungen“ (562). Das soll nicht prinzipiell, sondern nur so weit geändert werden, als Ungleichheit unangemessen ist. Das Kriterium für Einkommens- und Vermögensabstufungen ist aber nicht ein individueller Grenznutzen, sondern ein demokratisch ausgehandelter Gemeinnutzen. In diesem Aushandlungsprozess werden ,die gängigen Vorstellungen von ,gerecht“ und ,ungerecht““”(63) wichtig. Hier kommen die symbolische Aufladung und die moralische Wertigkeit von Ungleichheiten ins Spiel. Gerade deshalb argumentiert Piketty so vehement gegen die rhetorischen Rechtfertigungsfiguren eines ,,meritokratischen Extremismus“ (443), der Spitzeneinkommen und Ungleichheit mit besonderen Leistungen legitimiert, obwohl er bei näherem Zusehen auf nichts als auf arroganter Ignoranz gegenüber einem egalitären Demokratieverständnis basiert.

Es ist nicht zuletzt dieses Eintreten für eine Gesellschaft, in der sich Leistung lohnt, die Pikettys Argumente nach verschiedenen Seiten anschlussfähig machen. Denselben Effekt hat seine Aktualisierung der Makroökonomie. Nach einer ganzen Kaskade von wirtschaftswissenschaftlichen Paradigmenwechseln - von behavioral economics über evolutionary economics bis hin zu neuroeconomics - greift Piketty im „Kapital im 21. Jahrhundert" Formeln auf, die seit Ende der 1930er Jahre vorgeschlagen und während der fortschrittsgläubigen Nachkriegszeit zur Stabilisierung eines volkswirtschaftlichen Gleichgewichts weiter entwickelt wurden. Ein Vierteljahrhundert nach der Implosion des Ostblocks distanziert er sich zwar prononciert von der „Happyend“Stimmung, welche die Wirtschaftswissenschaft während des Kalten Krieges verbrei-

19 Tagesanzeiger, 13. April 2014: http://www.tagesanzeiger.ch/wirtschaft/konjunktur/Marx-wollte-absolute-Wahrheit--ich-nicht/story/31 433512?track (24.05.2016).

20 Vgl. auch: Thomas Piketty, A New Deal for Europe, in: The New York Review of Books, 25. 02. 2016, 4 (http://www.nybooks.com/articles/2016/02/25/a-new-deal-for-europe). 
tete ( $25 \mathrm{ff}$.). Doch weit davon entfernt, ein Abtrünniger der neoklassischen Theorie zu sein, spielt er deren Potenzial gegen ihre konzeptionelle Verödung durch Marktanbeter aus.

Piketty regt immer wieder dazu an, die „Entfaltung neuer Formen des Eigentums am Kapital und seiner demokratischen Kontrolle“ (782) voranzutreiben. Doch hat er nicht das Zeug zum Radikalen. Seine politischen Vorschläge erschöpfen sich - horribile dictu - in der Erfindung einer Steuer. In einer progressiven Kapitalsteuer, die auch das Problem der Vererbung großer Vermögen vermindern könnte, sieht er den wirksamsten Hebel, um die Macht des Kapitals auf rationale, demokratisch legitimierte Weise zu bekämpfen. Nils Minkmar kommentierte diesen Vorschlag mit den Worten: „Man kann darüber wehmütig werden: Die große Utopie unserer Generation ist eine neue Steuer?" ${ }^{21}$ Im sozialdemokratischen Problemhorizont Pikettys leuchtet diese Engführung auf eine fiskalische Forderung durchaus ein. Er sieht in Steuern demokratisch legitimierte Instrumente, die den Kampf gegen die Macht des Kapitals an rechtsstaatliche Normen zurückbindet.

Damit erklärt sich auch, wieso er seine „Wirtschaftsanalyse“ (31) nicht klassenkämpferisch zuspitzen, sondern demokratietheoretisch ausbauen will. Er plädiert insbesondere für die „Entwicklung einer multipolaren politischen Ökonomie“ (9), die Gleichungssysteme neu interpretiert als „Ungleichungen“ und sie auf diese Weise auf die kapitalistische Wachstumsdynamik und politische Spannungsfelder zu beziehen vermag. Eine solche Ökonomie zeichnet sich auch durch die Fähigkeit aus, Geschichten zu erzählen und Argumente narrativ zu strukturieren, was sich bei Piketty immer wieder eindrücklich zeigt. Solche Erweiterungen der Ökonomie können auch für die Geschichtswissenschaft Impulse freisetzen.

Es treten allerdings auch die Grenzen und Beschränkungen eines solchen Ansatzes hervor. Was die historisch-anthropologische Erforschung der Arbeit betrifft, so holt sich diese ihre Anregungen nach wie vor besser bei Marx als bei Piketty. Denn der Verfasser von „Das Kapital“ von 1867 regt einen analytisch geschärften Blick auf den Wandel der Arbeitsformen und der Definitionen dessen, was als „Arbeit“ zählt, an. Durch Marx inspirierte Theorietraditionen haben sich zudem in produktive Ansätze wie gender studies, postcolonial studies und environmental history eingeschrieben bei Piketty findet sich dazu wenig oder nichts. Gemessen am Problembewusstsein dieser Forschungsfelder erweist sich „Das „Kapital“ von 2013/14 trotz des Plädoyers für einen breiten, interdisziplinären Zugang als enttäuschend.

Was hingegen unverzichtbar bleibt, ist die Frage nach der Ungleichheit, die Piketty mit Marx verbindet und die viele Kritiker übersehen oder übergehen, so auch Hernando de Soto. ${ }^{22}$ Es gibt durchaus, wie de Soto feststellt, eine enorme Asymmetrie in der Verfügbarkeit von Daten. Der Vorwurf eines „Eurozentrismus in extremster Form“, den er gegenüber einem zum marxistischen Popanz aufgebauten Piketty übt, übersieht aber zunächst einmal die Vielfalt von Buchhaltungstechniken und Aufschreibesyste-

21 Nils Minkmar, Zu Besuch bei Thomas Piketty Der neue Star der Intellektuellenszene, in: Frankfurter Allgemeine Zeitung, 07.05. 2014: http://www.faz.net/aktuell/feuilleton/zu-besuch-bei-thomaspiketty-der-neue-star-der-intellektuellenszene-12 927888.html?printPagedArticle=true\#pageIndex_2 (24.05.2016).

22 De Soto, Pikettys armseliger Eurozentrismus. 
men rund um den Globus. Anstatt mit de Soto eine statistisch basierte Ungleichheitsforschung als ethnozentrisch zu diskreditieren, ist es nötig, die von starken Gefällen geprägte globale Informationstopographie und ihre Machteffekte in die Untersuchung miteinzubeziehen.

Auch Differenzierungen, Unschärfen und Überschneidungen in den Konzepten von Kapital und Arbeit gilt es neu zu denken. De Sotos Position ist hier polemisch-plakativ. Er schreibt, es gebe „für uns Nichtwestler [...] keinen Sinn, wenn er [Piketty, JT] Kapital als eine Substanz herausarbeitet, die den Interessen der Armen entgegenläuft“. Denn die Kämpfe und Kriege, die insbesondere im Nahen Osten und in Afrika stattfinden, seien „,keine Revolte der Armen gegen das Kapital, sondern Revolten um das Kapital“. In einem grossen Teil der „Dritten Welt“ würden Menschen „,weder Kapital noch Arbeit verkörpern, sondern eine Verbindung aus beiden“. Hier lässt sich von Piketty lernen, dass eine Generalisierung des Humankapital-Ansatzes wenig geeignet ist, um die von de Soto ins Zentrum gerückte ,unternehmerische Aktivität der Armen“ und ihre „Ambition, Teil des Weltmarkts zu sein“, zu analysieren. Vielmehr sollte die Proliferation kleiner „Entrepreneurs“ mit einem erweiterten Arbeitsbegriff untersucht werden, der eine binäre Unterteilung in „Unternehmer“ versus „Proletarier“ ebenso meidet wie einen bipolaren Schematismus „freie“ versus „unfreie Arbeit“. Am Pol der letzteren tauchten dann auch persistente Formen der Sklaverei auf, in denen sich das Kapital-Problem nochmals anders stellt. ${ }^{23}$ Jeder Versuch, eine Globalgeschichte der Arbeit zu schreiben, muss sich einfügen in eine Analyse globaler Machtstrukturen, kolonialer Herrschaftstechniken und internationaler Kapitalmärkte. Die Geschichte der Arbeit in Erweiterung kommt nur dann voran, wenn sie mit einer erweiterten Ungleichheitsforschung verbunden wird - und für letztere hat Pikettys Buch neue Schneisen geschlagen.

23 Vgl. dazu The Global Slavery Index: http://www.globalslaveryindex.org/ und den Report "The Global Slavery Index 2014": http://d1p5uxokz2c0lz.cloudfront.net/wp-content/uploads/2014/11/ Global_Slavery_Index_2014_final_lowres.pdf(24.05.2016). 